\title{
An approach to support entries for a design failure modes and effects analysis
}

\author{
Felicia Veronica Banciu $^{1, *}$ \\ ${ }^{1}$ Politehnica University Timişoara, Mechanical Faculty, IMF Department, 300222 Timişoara, \\ România
}

\begin{abstract}
The design of a fixture device is very laborious, requiring consideration of many issues from series production, information on the raw part material, required precision surfaces, ensuring an appropriate orientation scheme, continue with requirements related device itself as number of parts, parts type, complexity, manageability, easy operation and maintenance. That is why realising a proper analyse regarding the possible failures associated to these issues is overwhelming. This paper's aim is to propose an approach for collection of items necessary for starting a Design FMEA, using Functional Analysis, TRIZ functional modelling and axiomatic design approaches. The proposed approach - to extract items for a FMEA analyse - is applied to an example of an expanding mandrel regarding its internal components and theirs relations in order to accomplish the main requirement: the accurate locating and clamping of a part.
\end{abstract}

\section{Introduction}

There exists many tools, methods and approaches to manage the design process and some of them are general ones; they have general rules applicable in a wide range of products. This is also the case of FMEA which is an approach done by a cross multidisciplinary team and developed during the product and its associated processes design, it is a common approach where its applied and addressed elements are different but the logic is the same.

In first stages of product's design it is important to find ways to support and argument decisions making in order to avoid to recall some functions later in design process. It is also important "to see" what happens in case of a choice or another or to see what are the consequences of a failure to a certain component at a certain level of design, its implications in proper product functioning or its main functions achievement.

The function is the core element that "guides" the product's design and functional analysis is a common tool used for functions inventorying. A functional analysis helps to list product's internal and external functions but without emphasizing their harmful effects if they exist.

The design of a fixture device is very laborious, requiring consideration of many issues from series production, information on the raw part material, required precision surfaces,

${ }^{*}$ Corresponding author: felicia.banciu@upt.ro 
ensuring an appropriate orientation scheme, continue with requirements related device itself as number of parts, parts type, complexity, manageability, easy operation and maintenance. That is why realising a proper analyse regarding the possible failures associated to these issues is requiring a lot of time, multidisciplinary teams and could be subject to numerous failures.

This paper's aim is to propose an approach for collection of items necessary for starting a FMEA. The idea of the approach is to enrich the approach from [1,2] using Functional Analysis, TRIZ functional modelling and axiomatic design approaches, the latest for tracking the existing couplings and their impacts of propagation breakdowns.

\section{Approaches used in product's design}

\subsection{FMEA}

FMEA is a method that is applied at any detail level in design-component, function/subassemblies and assemblies and implies a very good understanding of systems functions and design and it implies the whole product design process [3].

To realize a FMEA following activities are done: an inventory of failure modes, what effects they have on the product, process and corrective measures that are to be applied. If analyse of failure criticity is followed, the RPN are calculated using existent data, existent reliability informations and external environment. They are very important and should be inventoried, the potential safety implications in system failures, its performance indicators, maintainability indicators extraction and not least the generation of demands for maintenance service, assembly, packaging, materials, safety, service operation. It is essentially an evaluation technique that can be applied at any level of detail following an approach as well as bottom-up or top down up. It applies equally to functions, subsystems, parts and assemblies, as a basic requirement without which cannot be achieved a good functions understanding and system operation. FMEA can be applied to recognize probable failure modes, conclude their effect on the process of the product, and categorize actions to diminish the failures. A vital step is anticipating what might go incorrect with a product. It is a popular tool for reliability and failure-mode analysis [4]. Process FMEA is described as having the goal to identify failures associated with the fabrication, assembly processes, machine tools, fixtures and Production Methods. Design FMEA deals with possible components failure; consider the integration of components and their interactions effects on product functions, subassemblies functions $[3,5]$.

\subsection{Functional analysis}

FAST - tool used for functional analysis-is a diagram that facilitates graphically and logic view of what functions are and how they work together. Thinking a product in terms of functions does not hinder / constrain thinking related to the physical / processes features and characteristics and therefore can lead to a better problem definition and an easier way to solution. FAST describes the logic relations that must exist between the various functions that must be met in order to satisfy the main product's functionality [6]. FAST diagrams and thinking is the base for different studies, approaches or technical analyses.

\subsection{TRIZ functional modeling}

Related to product's functions also functional TRIZ modeling [7, 8] could be used. The HU Diagrams (Harmful-Useful Diagrams) are designed around the concept that all systems 
have positive aspects (useful functions) and all systems have negative aspects (harmful functions). A function is defined as capturing some aspect of a system including function, activity, state, process, condition, transformation. There are eight possible interactions/links between-useful-harmful functions ones producing or counteracting another.

\subsection{Axiomatic design framework}

The design is interplay between what we want to achieve and how we choose to satisfy the need. To systematize the thinking process in this interplay, it was created the domain concept, foundation of axiomatic design and it is guided by two axioms. These axioms are: the Independence Axiom - maintain the independence of the functional requirements (FRs) sections of the design should be separable so that changes in one have no (or as little as possible) effect on the other and the Information Axiom - minimize the information content of the design. The four domains are: Customer Domain, Functional Domain, Physical Domain and Process Domain and for each domain there are corresponding Customer Needs (CNs), Functional Requirements (FRs), Design Parameters (DPs) and Process Variables (PVs). The relations between the functional requirements FRs and design parameters DPs at a given level of the design hierarchy are captured in the Design Matrix [9]. The axiomatic design methodology is based on functional analysis and minimization of information content - aiming a robust design $[9,10]$.

\section{The proposed approach}

The general view of proposed approach is in figure 1 .

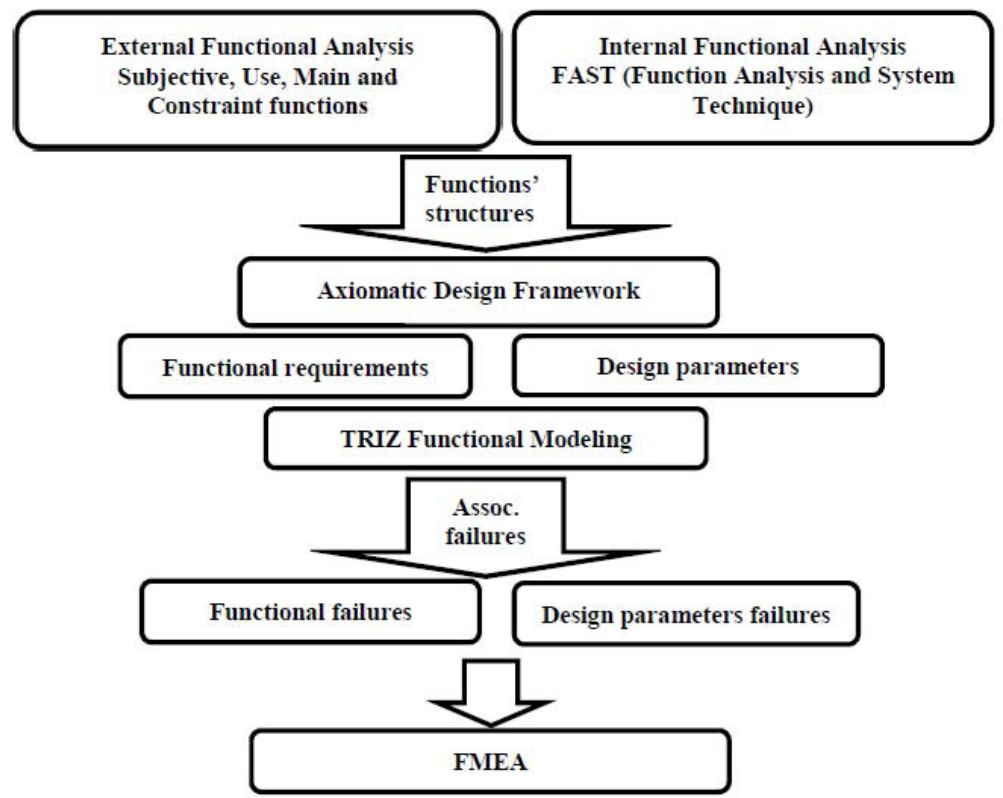

Fig. 1. The proposed approach.

As a work procedure/approach, first applying a functional analysis - the technical functions and elements that conducts to achieve the main functions, also the external environment elements will emphases the functional links and flows.

The results of a functional analysis - identification of functions - are entries in 
Functional Requirements domain and for all of these functions are inventoried the physical parts/subassemblies which accomplish these functions - the design parameters (axiomatic design).

The design matrix contains the FRs-DPs elements. Also here are emphasised the eventual couplings that exist between design parameters. A framework to systematize and have an overall view on functions/components/potential failures and their consequences on different detail levels could be that given by axiomatic design.

The possible couplings will allow following the failure modes track, namely a failure at a certain level can influence occurrence of failures to lower levels of detail or on the same level. In this paper is presented the overall approach without a deep detailed view on each of its steps-design methods/tools.

A functional analysis helps to list product's internal and external functions but without emphasizing their harmful effects if they exist and to solve this, applied to functional requirements and design parameters a TRIZ functional modeling could be useful. We can obtain a complete function structure regarding the useful and harmful function effects if the TRIZ functional modeling is applied to those functions identified through a functional analysis. Thinking in terms of functional modeling, for these functions there can be identified their useful and harmful effects associated to these will be inventoried and possible useful or harmful effects of design parameters. This will represent a part of the entry data needed for a FMEA. In this paper is presented only the general approach without a complete modelling technique that deals only with useful-harmful functions and their effects on each other, this being a subject for further work. In addition, the design matrix associated to FR-DP is not represented as a matrix but the couplings between design parameters are mentioned and explained.

\section{Proposed approach applied to a self-centring fixture device}

Fixtures are considered as a subsystem with a functional independent activity that provides accurate locating and clamping for part during the machining operations. The acting elements that intervene in design process and generates inputs and constraints are:

a) external elements

- part material class, type, properties; part configuration (Number and dimension of holes, surface types, symmetry, slots, bosses etc. ), shape, size, -tolerances (linear and angular) and accuracy, roughness, surface coatings,

- machine tool (machine tool type (its equipment and also its characteristics), interference check (machine tool has to be able to perform all movements with fixture, tool and part in their places ) Forces and moments acting during machining process, chips removal,

- cutting tool-operator (accident proof concept, fool-proof concept, ease of use, loading and unloading efficiency),

- manufacturing process regarding the operations (machining) on parts, - type of machining, number of machining surfaces (single surface, more identical surfaces in linear displacement, more identical surfaces in circular displacement);

- batch size.

b) internal elements:

- a great importance should be spent on clamping device itself, namely on its internal components and theirs relations in order to accomplish the main requirement: accurate locating and clamp of a part.

The proposed approach will be applied to an example of expanding mandrel (figure 2) regarding the internal components and theirs relations in order to accomplish the main requirement: accurate locating and clamp of a part. The functions inventoried during an internal functional analysis correspond to each of the mandrel parts or subassemblies roles. 
This expanding mandrel in figure 2 is used for centring and fixing an inner cylindrical part. It is centred and fixed using the disk jaws 2 displaced in axial movement by bushing 8 . This bushing is mounted on stem 9 using a retaining 6 and locking nut 5 and thus is made the link with the actuating/operating system. The disks (plate fin) 2 are radially displaced and guided using the multiple wedge 4 . Springs with ring shape located in disks slots assure the contact between disks and wedge. To avoid the rotation of bushing 8 against the wedge is used the sliding key 7. Centring and fixing the part is done actuating the stem in left direction and for loosening the part in the right direction.

Against the part are working radial forces $\mathrm{S} / \mathrm{n}$ and in the same time, there appear frontal forces - a good axial part positioning and friction torque.

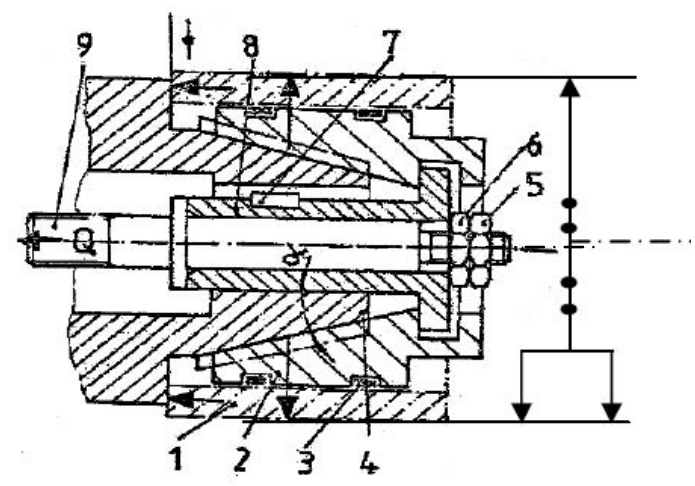

Fig. 2. Expanding mandrel [11].

Analysing the mandrel operation and its components and applying the Internal Functional Analysis FAST (Function Analysis and System Technique) (first part from approach in figure 1) were identified the functional requirements. These functions generates the functional requirements entries and their associated design parameters (way of their achievement) that are the base of the axiomatic design approach.

Next, using the TRIZ modeling technique and thinking from a useful/harmful perspective the functional requirements and their associated design parameters it were analysed the possible positive and negative aspects. All of these functional requirements and theirs associated design parameters- existent solutions and parts - have attached the useful-harmful perspective with their associated useful and harmful aspects are presented in table 1. This table offers an overview on possible issues and harmful/negative aspects that are main entries in a Design FMEA analyse regarding the possible failure modes.

Table 1. Functional requirements and design parameters - useful and harmful aspects.

\begin{tabular}{|l|l|}
\hline \multicolumn{1}{|c|}{ Functional requirements FRs } \\
\hline $\begin{array}{l}\text { FR0 Centring and hold the WP on its inner } \\
\text { cylindrical surface } \\
- \text { useful function, useful effect }\end{array}$ & DP0 Self-centring mandrel \\
\hline $\begin{array}{c}\text { FR1 Centring WP on inner cylindrical } \\
\text { surface } \\
\text { - useful function }\end{array}$ & DP1 Materialize WP axis \\
\hline $\begin{array}{c}\text { FR11 Use up clearance between work } \\
\text { piece and elements }\end{array}$ & DP11 Elements that contact the WP \\
\hline $\begin{array}{c}\text { - useful function, useful effect } \\
\begin{array}{c}\text { FR11 Contact between the work piece } \\
\text { and centring/fixing elements } \\
\text { - useful function with useful and harmful } \\
\text { effects }\end{array}\end{array}$ & $\begin{array}{l}\text { DP111 Disks } \\
\text { - Darmful effects : } \\
\text { - Contact friction }\end{array}$ \\
\hline
\end{tabular}


It implies that work piece to be in contact with disks in order to receive the force- useful function

FR12 Transmit the movement synchronous to disks

- useful function

- useful function
FR121 Disks movement

DP12 Mechanism with actuation/operation rod/stem

- one harmful effect is the fact that the stem is pulled and has to be tensile-

DP121 Multiple wedge with sloped surfaces in contact with disks

Harmful effect

- friction forces and wear

FR1211 Contact between multiple wedges and disks

- useful function

- the contact between multiple wedges and disks implies surface wear and friction issues

DP1211 elastic force

- The disks under the spring's elastic force has to move so a harmful effect here can be introduced by friction force that has to be lower;

- Another negative aspect here can be that the springs lose their properties, the improper elastic force developed by springs.

DP 1212 ring type springs

DP 1213 slots for ring springs in wedges body

FR1212 Guide disks in radial movement

DP1212 Guiding slots on surface of multiple wedge

- it implies that two pieces to be connected in order

allow the movement;

- the contact area, the type of fit between disks and wedge
FR 13 Moving disks on sloped surfaces of multiple wedge

- useful function

DP13 Operating disks, using a bushing mounted on stem/rod

- it implies that two pieces to be connected in order to transmit the movement. This raises two aspects that have to be considered:

- the contact area bushing and disks

- the fact that the stem is pulled and has to be tensile

FR131 Transmitting the operating force to bushing

DP 131 retaining nut and safety nut mounted on screw stem/rod
Harmful effects:

- Contact area between safety nut and retaining nut

- Contact area between nuts and stem

- Contact between retaining nut and bushing

- Wear issues related with Contact between retaining nut and bushing

DP 1311 close fit between bushing and stem

- contact pressure assures the force and movement transmitting and in the same time there could be a harmful effect related to high pressure in contact area

DP 132 Bushing shape

Harmful effect

- contact area-friction, wear

DP 133 Free fit between bushing and multiple wedge

FR 133 Multiple wedges sliding against stem/rod and bushing
FR 134 Avoid bushing rotation against multiple wedges

- useful function

DP 134 Using a sliding key

Harmful aspects

- Contact between sliding key and bushing

- Contact pressure

- Wear and friction between sliding key and its guiding slot in multiple wedge

FR15 transmit the movement to the operation stem
DP 15: operator/machine tool 


\begin{tabular}{|l|l|}
\hline $\begin{array}{l}- \text { useful function -harmful effect is the fact } \\
\text { that the stem is pulled and has to be tensile }\end{array}$ & DP 2 provide the holding forces \\
\hline $\begin{array}{l}\text { FR2 prevent the WP movement during } \\
\text { working }\end{array}$ & DP21 wedges functional characteristics \\
\hline FR21 self - locking mechanism & $\begin{array}{l}\text { DP22 the mechanism with wedges and plungers, } \\
\text { provide F*n force } \\
\text { friction forces that has both positive as negative } \\
\text { aspects }\end{array}$ \\
\hline $\begin{array}{l}\text { FR22 prevent WP rotation } \\
\text { - Useful function }\end{array}$ & DP23 WP propped in mandrel's body and its shape \\
\hline FR3 loosen, un holding the WP & DP3 assure the reverse movement \\
\hline $\begin{array}{l}\text { FR 31 transmit the reverse movement to } \\
\text { the operation rod/stem }\end{array}$ & DP 31 machine tool \\
\hline FR 32 reverse disks movement & DP32 operating stem and bushing in the right side \\
\hline FR33 Disks inward movement & DP33 elastic force, springs \\
\hline
\end{tabular}

In this table, in the first column are functional requirements achieved using one or more than one design parameter. In this second case when a functional requirement is achieved using more than one design parameter, the line containing these design parameters has its correspondent first column blank in functional requirements place.

The physical contact between mandrel's parts generates friction forces that has both useful and negative aspects. In addition, aspects related to surfaces characteristics, surface wear, stress could conduct to failure and could be considered as entrance elements in analysing possible failure modes. In axiomatic design (and in designing, generally), the link between functional requirements (what is needed) and design parameters (solutions) are given by mathematical equations expressing their dependencies.

The contact between the work piece and centring/fixing elements is a function that has both useful and harmful effects - useful is that friction helps part fixture but also it has to be considered the contact area between parts - the wear and contact issues are the harmful effect.

The force $\mathrm{F}$ depends on the value of the wedge's parameters- angle, sliding angles between wedges and disks, friction coefficients between all the parts being in relative movement. Even if the design is functional from a mathematical point of view, with imposed physical parameters, it can happen that force F cannot be achieved due to other components e.g. springs marked on Fig. 2 with 3. If is considered the FR121 with its DP 121 , the corresponding sub-matrix is uncoupled, not dependent on other assemblies (it is about a solution to keep together the disks against the multiple wedge) but the fail generated by springs is major because it leads to not achieve the FR0, higher level requirement. The design parameter for example "guiding slots on surface of multiple wedge" does not appear directly in calculating the force F but its fail (the contact area, the type of fit between disks and wedge) can affect force. Taking into account that this expanding mandrel is with moving elements, its accuracy can be discussed relatively to another types of mandrels proper to deliver the main functions. This is another negative aspect.

\section{Conclusions}

The advantage of applying this approach is the fact that splitting the product (device) into functions and their associated parts/subassemblies and thinking to their useful and harmful effects offers a deeper and detailed view on whole device/product from different point of views - functional/parts/effects- and could be used as entries for a Design FMEA. 
In future work the presented approach could be continued through a complete TRIZ modelling technique that deals only with useful-harmful functions and their effects on each other and with design matrix. All the methods/tools presented in approach need a thorough teamwork.

The approach presented in this paper is a general one and its novelty and originality are that it could be adapted to various designs being useful in systematizing the knowledge about functional role/functions/components/process/useful-harmful sides of functions/components used as entries in analysing of potential failures modes and their causes. Analysing the harmful functions effects will offer a broader perspective of issues related to fixture design and associated failure modes thus leading to improvements, to discover failure at its earliest possible point in product design process. Simultaneously, broadening the perspective on failure modes issues, it can be a support for continuous innovation.

\section{References}

1. F. Banciu, G. Drăghici, A New Functional Approach to Support FMEA Method in Product Conceptual Design, in Proceedings of the $16^{\text {th }}$ International Conference Modern Technologies, Quality and Innovation, 1, (2012)

2. F. Banciu, G. Draghici, C. Turc, A Point of View on Functional Approaches Used in Product Design in Applied Mechanics and Materials 371, (2013)

3. Failure Mode Effects Analysis (FMEA) at http://asq.org/learn-about-quality/processanalysis-tools/overview/fmea.html

4. L. Lipol, J. Haq, Risk analysis method: FMEA/FMECA in the organizations. Int. J. of Basic \& Appl. Sci. IJBAS-IJENS 11, 74, (2014)

5. S.-H. (Gary) Teng, S.-Y Ho, Failure Mode and Effects Analysis: An integrated approach for product design and process control. Int. J. of Quality \& Reliability Management 13, 12 (1996)

6. J. Borza, FAST Diagrames: The Foundation for Creating Effective Function Models, Trizcon 2011, 1-10, (2011)

7. J. Terninko, A. Zusman, B. Zlotin, Systematic Innovation: An Introduction to TRIZ (Theory of Inventive Problem Solving), (Saint Lucie Press, Boca Raton, Florida, USA, 1998)

8. S. D. Savransky, Engineering of Creativity: Introduction to TRIZ Methodology of Inventive Problem Solving, (CRC Press 2009)

9. N. P. Suh, "Axiomatic Design-Advances and Applications", (New York, Oxford University Press, 2001)

10. D. G. Lee, N. P. Suh, Axiomatic Design and Fabrication of Composite Structures, (Oxford University Press, 2006)

11. N. Gojinețchi, N. Gherghel, Workholding Devices Design (Iasi, 1983) 